\title{
Upregulation of p18Ink4c expression by HPV E6 via p53-miR-34a pathway
}

\author{
Xiaohong Wang ${ }^{1 *}$, Craig Meyers ${ }^{2}$, Zhi-Ming Zheng ${ }^{1}$ \\ From $12^{\text {th }}$ International Conference on Malignancies in AIDS and Other Acquired Immunodeficiencies \\ (ICMAOI) \\ Bethesda, MD, USA. 26-27 April, 2010
}

Binding of p53 to miR-34a promoter activates the expression of tumor suppressive miR-34a. Our previous study demonstrated that oncogenic HPV infection downregulates miR-34a expression through degradation of p53 mediated by E6. In searching for miR-34a targets, we found that miR-34a mainly downregulates p18Ink4c, a CDK4/6 inhibitor induced by E2F transactivation. HPV18+ HeLa cells with ectopic miR-34a expression or by E6 siRNA knockdown-induced expression of miR-34a had a substantially reduced expression of p18Ink4c in a dose-dependent manner, but had no effect on p16Ink4a, another member of CDK4/6 inhibitor family. Further investigation showed an increased p18Ink4c level in cervical cancer tissues by western blotting as compared to normal cervix. By immunostaining of tissue arrays, an increased expression of p18Ink4c was found in $68 \%$ of cervical cancer tissues, but only in $6.6 \%$ of normal cervical tissues. Moreover, specific inhibition of miR-34a expression in CaSki cells promoted the expression of p18Ink4c. Raft cultures with HPV16 or HPV18 infections also showed an increased p18Ink4c expression. In a reporter assay, we demonstrated that disruption of the miR-34a seed match from p18Ink4c promoted luciferase activity in the presence of ectopic miR-34a. As a cell cycle regulatory protein in normal cells, the increased p18Ink4c expression by E6-induced p53 destabilization and miR-34a reduction in cervical cancer would lead to suppress cell cycling and proliferation. However, viral E7 expressed in cervical cancer cells inactivates $\mathrm{pRB}$ and disassociates E2F from $\mathrm{pRB}$, resulting in disruption of normal G1 checkpoint. Thus, an increased p18Ink4c in the cervical cancer cells would not affect the cell growth.
To test this hypothesis, we knocked down p18Ink4c expression by siRNA in both HPV18-positive HeLa cells and HPV-negative HCT116 cells, a colon cancer cell line that contains an intact $\mathrm{G} 1$ checkpoint. As we predicted, knocking down p18Ink4c expression promoted the growth of HCT116 cells but had no effect on HeLa cells. Altogether, our data indicate that p18Ink4c is a miR-34a target and its increased expression in cervical cancer tissues can be used as a new biomarker for cervical cancer diagnosis and prognosis.

\section{Acknowledgements}

This article has been published as part of Infectious Agents and Cancer Volume 5 Supplement 1, 2010: Proceedings of the $12^{\text {th }}$ International Conference on Malignancies in AIDS and Other Acquired

Immunodeficiencies (ICMAOI). The full contents of the supplement are available online at http://www.biomedcentral.com/1750-9378/5?issue=S1.

\section{Author details}

'HIV and AIDS Malignancy Branch, Center for Cancer Research, National Cancer Institute, National Institutes of Health, Bethesda, MD, USA.

${ }^{2}$ Department of Microbiology and Immunology, Penn State University School of Medicine, Hershey, PA, USA.

Published: 11 October 2010

doi:10.1186/1750-9378-5-S1-A85

Cite this article as: Wang et al:: Upregulation of p18Ink4c expression by HPV E6 via p53-miR-34a pathway. Infectious Agents and Cancer 2010 5(Suppl 1):A85.

\footnotetext{
*Correspondence: wangxiah@mail.nih.gov

${ }^{1}$ HIV and AIDS Malignancy Branch, Center for Cancer Research, National

Cancer Institute, National Institutes of Health, Bethesda, MD, USA

Full list of author information is available at the end of the article
} 